\title{
Tuned diamond turning of micro-structured surfaces on brittle materials for the improvement of machining efficiency
}

\author{
Zhiwei Zhu a, Zhen Tong b, Suet To (2) c, ${ }^{*}$, Xiangqian Jiang (1) ${ }^{b}$ \\ a. School of Mechanical Engineering, Nanjing University of Science and Technology, Nanjing, 210094 JS, China \\ b. EPSRC Future Metrology Hub, Centre for Precision Technologies, University of Huddersfield, Huddersfield, UK HD1 3DH \\ c. State Key Laboratory of Ultra-precision Machining Technology, Department of Industrial and Systems Engineering, The Hong Kong Polytechnic University, Kowloon, \\ Hong Kong Special Administrative Region, China
}

Target at improving the machining efficiency for diamond turning of micro-structured surfaces on brittle materials, a general technique named tuned diamond turning is proposed through strictly matching the maximum depth-of-cut with the critical one during turning, and the corresponding toolpath planning algorithm is developed. Practically, a novel piezo-actuated dual-axial fast tool servo system is introduced to realize the tuning concept, and a typical micro-structured surface free from cracks is successfully generated on a single crystal silicon wafer. Compared with fast-/slow tool servo, the machining efficiency is improved by a factor of $16.35 \%$, demonstrating the effectiveness of the tuned diamond turning.

Cutting; Brittleness

\section{Introduction}

Although it is very challenging for the processing of brittle materials with low fracture toughness, the fast/slow tool servo (F-/STS) diamond turning technique has attracted everincreasing attentions to generate freeforms and micro-structured surfaces on brittle materials, attributing to its high accuracy and high flexibility [1].

With the face turning of a planar surface using a roundly edged tool, the maximum depth-of-cut is jointly dependent on the tool nose radius, the nominal depth-of-cut, and the radial feedrate [2]. To avoid cracks on the machined surface, the most direct way is to remove the material in a totally ductile mode. It can be achieved by making the maximum depth-of-cut be strictly no larger than the critical depth-of-cut which is defined as the depth for the material at which the brittle and ductile transition occurs [3]. An alternative way to get the crack-free surface is to cut with controlled cracks using a slightly larger feedrate, and the cracks must be capable to be removed in the following cutting passes [4]. Although the critical depth-of-cut varies with respect to material property and tool geometry [2], it is normally in the submicrometer range, requiring an extremely small feedrate to finally get a crack-free surface [5]. In common, the small allowable feedrate may significantly restrict the machining efficiency. Therefore, much effort has been devoted to achieving cost-efficiently machining of brittle materials.

As the critical depth-of-cut is an essential factor dominating the machining efficiency, the attempt to achieve a larger critical depth-of-cut has been widely performed, which are mainly based on two aspects, namely the material property modification and the external high energy assistance in cutting. In general, the ion implantation based material modification has been reported to be beneficial for the increase of the critical depth-of-cut through introducing the phase transformation layers [6]. Meanwhile, the high energy assisted cutting including the laser based thermal energy [7] and the vibration based mechanical energy [8] has also been demonstrated to be effective to get larger critical depth-of- cuts for a wide spectrum of brittle materials through enhancing the material plastic flow during cutting.

Taking advantage of those methods, the critical depth-of-cut can be effectively increased to have a remarkable improvement of the machining efficiency for the generation of freeforms [6] and micro-structured surfaces [1] through F-/STS. However, no matter which method is adopted nowadays, the shape feature of the desired micro-structured surface is commonly ignored, and a small enough feedrate for the largest depth-of-cut of a surface is commonly required to guarantee the ductile mode cutting for the whole process [4]. With the increasing complexity of freeforms and micro-structured surfaces, the practical depth-of-cut may significantly vary from position to position, and the currently adopted determination strategy for the feedrate is therefore over conservative for cutting positions with smaller depth-of-cuts, which becomes the bottleneck for the improvement of the machining efficiency of turning brittle materials.

To overcome this general limitation, a tuned diamond turning technique is proposed to maximize the feedrate for each cutting point with full consideration of the desired surface shapes. It is a universal method and can be easily combined with any aforementioned methods to further improve the machining efficiency.

\section{The dual-axial fast tool servo}

To tune the maximum depth-of-cut, an extra oscillation along the radial direction is essentially needed. Therefore, a novel dualaxial fast tool servo (DA-FTS) system is introduced to practically realize the tuning concept. Although a few DA-FTSs were reported for the active control of tool marks on the machined surface, those systems may suffer from low stiffness, low bandwidth, and insufficient motion accuracy [9]. Therefore, taking advantage of piezoelectric actuators (PEA) and compliant mechanisms, a new DA-FTS system with parallel configuration is specially constructed to have high bandwidth servo motions along the $x$ - and $z$-axial directions of the machine tool for the efficient generation of micro-structured surfaces. 
The designed mechanical structure for the DA-FTS is illustrated in Fig. 1 (a). In this design, two PEAs (PI P-887.51, Germany) are orthogonally arranged to generate the dual-axial motion, and four typical parallelogram flexure mechanisms are adopted with each consisting of two compliant linkages. From the principle shown in Fig. 1 (b), when one PEA expands, the parallelogram mechanism along its axial direction will deliver the motion to the end-effector where the diamond tool is attached. Meanwhile, the two sets of parallelogram mechanisms perpendicular to the actuation axis will guide the actuation motion for the end-effector, and the undesired parasitic rotations can be constrained by means of the parallelogram structure. The spring steel material is used to construct the DA-FTS, and the dual-axial motions are captured by two capacitive sensors from MicroSense cooperation (probe 5503 and charge amplifier 5810).

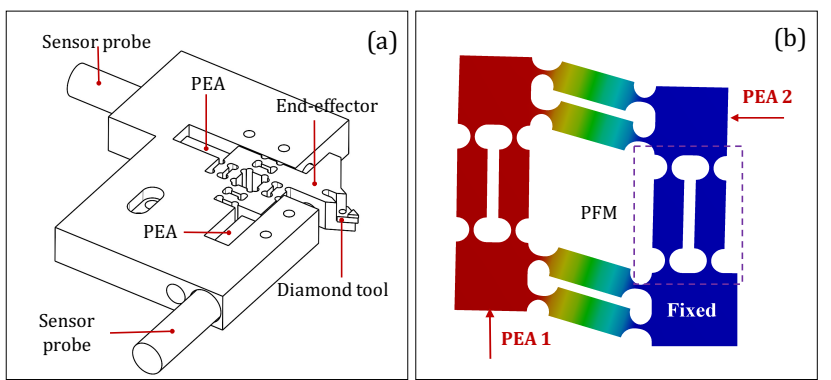

Figure 1. (a) Mechanical structure of the DA-FTS, and (b) its working principle, where PFM is short for the parallelogram flexure mechanism.

With the trajectory tracking, the integral-type sliding mode controller (I-SMC) featuring chatter free and zero steady state error is designed for the motion control along each direction [10]. The cross-axis coupling, system nonlinearities, and external forces are treated as lumped disturbances on the control system, which will be comprehensively compensated by the highly robust I-SMC. To implement the control system, the real-time control through the MATLAB/Simulink desktop real-time modulus is conducted via a data acquisition board (NI PCI-6529, USA).

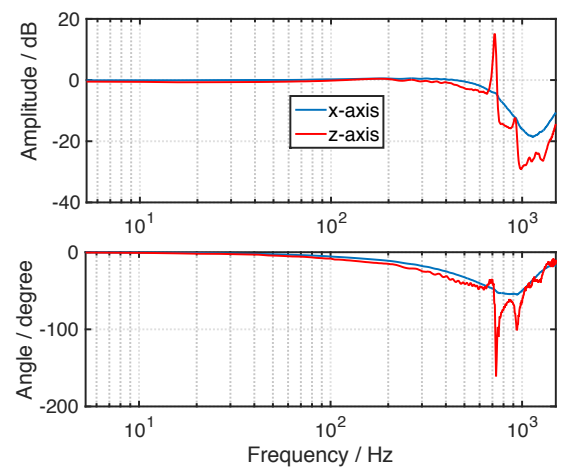

Figure 2. The frequency response functions for the closed-loop DA-FTS.

Through independent quasi-static testing and sweep excitation, the stroke and the first resonant frequency along the two directions are measured to be almost identical as $8.5 \mu \mathrm{m}$ and 3.5 $\mathrm{kHz}$, respectively. For the closed-loop system, a sweep excitation is performed for each actuation axis, and the corresponding closed-loop frequency response functions are illustrated in Fig. 2. The response function curve is observed to be relatively flat in the low-frequency region, and the working bandwidth $(-3 \mathrm{~dB})$ along the $x$ - and $z$-axial directions is identified to be $652 \mathrm{~Hz}$ and $530 \mathrm{~Hz}$, respectively. It is noteworthy that an obvious resonance is observed around $700 \mathrm{~Hz}$ for the $\mathrm{z}$-axial servo motion which is mainly caused by the non-ideal fix of the DA-FTS on the machine tool. A stiffer support is suggested for the installation of the DAFTS to avoid any low frequency resonances.

\section{Toolpath for the tuned diamond turning}

Unlike the toolpath planning for F-/STS which only considers the form accuracy of the machined surface, an active tuning of the practical depth-of-cut using an extra oscillation along the $x$-axial direction makes the toolpath planning more complicated for the tuned diamond turning. Considering the constraint of both the form accuracy and the accordance between the maximum and critical depth-of-cut, the toolpath planning algorithm for the tuned diamond turning is developed together with the algorithm for the tool clearance constraint.

\subsection{Toolpath planning algorithm}

Through the constant angle based discretization, the rotation angle in one revolution is evenly divided into $N$ pieces, and the rough surface is assumed to be planar. As shown in Fig. 3 (a), a fixed cylindrical coordinate system is constructed as $o-\rho \theta z$ for the machine tool with its $o z$ axis being coincide with the spindle axis, and it can be mapped into the corresponding Cartesian system $o$ $x y z$ by setting $x=\rho \cos \theta$ and $y=\rho \sin \theta$.

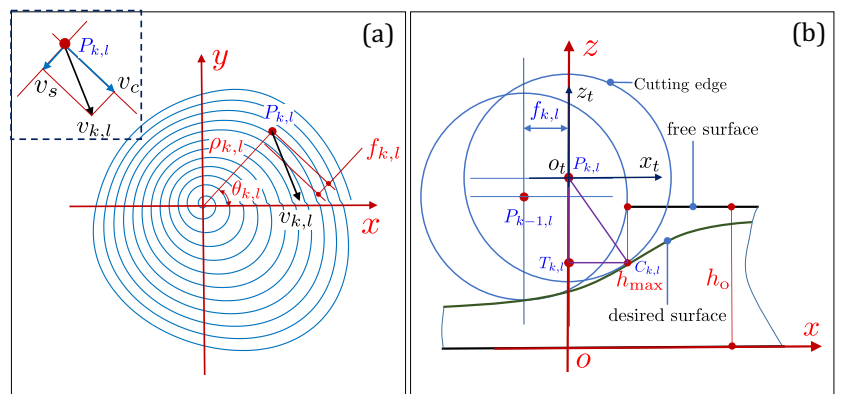

Figure 3. Toolpath for the tuned diamond turning, (a) the projected toolpath on the $x y$ plane, and (b) the cross-sectional view on the $x z$ plane. Since $h_{0}$ is much smaller than $r_{\varepsilon}$, the point $C_{k, l}$ which is on the cutting edge with maximum depth-of-cut can be assumed to be vertically below the intersection point between the free surface and tool edge at $P_{k-1, l,}$.

The cross-sectional drawing of the tool position along the radial direction in the $o-x z$ plane is further illustrated in Fig. 3 (b). It indicates that the maximum depth-of-cut $h_{\max }$ for the $l$-th tool center point (TCP) $P_{k, l}$ in the $k$-th revolution depends not only on the factors as discussed in Ref. [3] for planar cutting, but also on the desired surface shape as well as the successive tool position in the previous revolution, namely the position of $P_{k-1, l}$.

As illustrated in Fig. 3 (b), by projecting $C_{k, l}$ to a vertical axis crossing $P_{k, l}$, the geometry relationship in $\triangle P_{k, l} C_{k, l} T_{k, l}$ may lead to

$d_{t c}=\sqrt{r_{\varepsilon}^{2}-d_{t p}^{2}}$

where $r_{\varepsilon}$ is the tool nose radius, $d_{\mathrm{tc}}$ and $d_{\mathrm{tp}}$ are the distances from point $T_{k, l}$ to point $C_{k, l}$ and $P_{k, l}$, respectively.

From the geometry in Fig. $3(\mathrm{~b})$, the distance $d_{\mathrm{tp}}$ and feedrate $f_{k, l}$ can be obtained as

$d_{t p}=r_{\varepsilon}+z_{k, l}-\left(h_{o}-h_{\max }\right)$

$f_{k, l}=\sqrt{r_{\varepsilon}^{2}-\left(r_{\varepsilon}+z_{k-1, l}-h_{o}\right)^{2}}-d_{t c}$

where $h_{\mathrm{o}}$ is the nominal depth-of-cut, $z k, l$ and $z k-1, l$ are the $z$-axial servo motion for the TCP $P_{k, l}$ and $P_{k-1, l}$, respectively. As a critical state for ductile removal, the maximum depth-of-cut $h_{\max }$ could be replaced by the critical one $h_{c}$ in Eq. (2) to establish a governing law relating the cutting parameters and the material property.

From Eq. (1) to Eq. (3), there are two unknown variables $\left(f_{k, l}\right.$ and $z k, l)$ in one relationship $\left(h_{\max }=h_{\mathrm{c}}\right.$ ) to iteratively estimate the position of point $P_{k, l}$ from the previous point $P_{k, l-1}$. To determine 
the two unknown variables, another equation considering the tangency condition between the tool edge and surface shape is established through the numerical calculation method.

Assume that $o_{t}-X_{t} Z_{t}$ is the local Cartesian coordinate system of the diamond tool with $o_{t}$ being coincide with $P_{k, l}$ as shown in Fig. 3 (b). Through evenly dividing the tool edge into $2 N_{\mathrm{t}}$ pieces along the $o_{t} x_{t}$ direction, the $z_{t}$-axial profile of the edge can be expressed by

$$
z_{i}^{(t)}=-r_{\varepsilon} \sqrt{1-\left(i N_{t}^{-1}\right)^{2}}, i \in\left[-N_{t}, N_{t}\right] \in Z
$$

To simplify the calculation process, the tangency condition is equivalently modeled as the minimum approaching distance of the tool from a faraway position to exactly contact the surface to be machined. Therefore, from the constraint of form accuracy, another equation relating the two variables, i.e., $f_{k, l}$ and $z k, l$, can be mathematically expressed by

$$
z_{k, l}=\min \left|z_{o}+z_{i}^{(t)}+S\left(\rho_{k-1 . l}+f_{k, l}+i N_{t}^{-1} r_{\varepsilon}, \theta_{k-1, l}\right)\right|-z_{o}, \forall i \in\left[-N_{t}, N_{t}\right]
$$

where $z_{s}=S(\rho, \theta)$ denotes the $z$-axial surface coordinate at a point $(\rho, \theta)$ in the machine tool system, and $z_{0}>0$ is a virtual distance.

By solving these equations, positions of the TCPs for the whole turning can be iteratively obtained with full consideration of the desired surface shape, tool geometry, and the critical depth-of-cut of the material. Herein, the first TCP is placed at the center of the spindle, and a constant feedrate is adopted for the first revolution to provide the basic data for the following iterations. Although the first TCP can not be exactly at the center, the sub-micrometer tool setting error will not change the maximum depth-of-cut due to the fixed relative positions among the TCPs.

\subsection{Tool clearance constraint}

In the tuned diamond turning, the radial oscillation will cause a remarkable change of the cutting direction as illustrated in Fig. 3 (a). To avoid the interference between the tool clearance and the surface to be turned, the angle for the cutting direction change should be smaller than the tool clearance angle $\kappa$. Assume that the contact area at the tool edge is much smaller than the nose radius during turning, the cutting clearance can be regarded as the same, irrespective to the tool clearance type. Therefore, the constraint of the clearance angle can be expressed by

$\kappa \geq \max \arctan \left(\frac{\rho_{k, l}-\rho_{k, l-1}}{2 \pi \rho_{k, l-1}} N\right), \forall k, l$

From the viewpoint of tool motion, this relationship also gives a constraint for the increment of the $x$-axial oscillation with respect to a specified tool with a fixed clearance angle $\kappa_{0}$, namely

$$
\Delta_{k, l}=f_{k, l}-f_{k, l-1} \leq \frac{2 \pi}{N} \tan \left(\chi \kappa_{o}\right) \rho_{k, l-1}
$$

where $\chi \in[0,1]$ is a safety factor considering any uncertainties related to the nominal clearance angle of the diamond tool.

Note: As the radial oscillation is jointly determined by the desired surface shape and the critical depth-of-cut of the material, it would be possible that the radial oscillation may go beyond the constraint in Eq. (7) for certain conditions. If so, a scaling method for the decrease of the increment from $f_{k, l-1}$ to $f_{k, l}$ should be adopted to avoid undesired clearance interference.

\section{Tuned diamond turning results and discussion}

The turning experiment is performed on a three-axis ultraprecision lathe (Moore Nanotech 450 UPL, USA), and a single crystal silicon wafer is employed as the workpiece for this demonstration work. A conical clearance natural diamond tool (Contour fine tooling, UK) with a clearance angle of $12.5^{\circ}$ and zero rake angle is employed, and its nose radius is $r_{\varepsilon}=0.1 \mathrm{~mm}$. During turning, the wafer is glued to the end-face of a flat cylindrical brass bar which is fixed on the spindle through the vacuum chuck. The wafer is firstly flatted with a spindle speed of $2000 \mathrm{rpm}$ using the same cutting tool, and the nominal depth-ofcut and feedrate are set as $h_{0}=1 \mu \mathrm{m}$ and $f=0.2 \mu \mathrm{m} / \mathrm{rev}$ for the flatting, respectively. Under this condition, the maximum depthof-cut is calculated to be about $h_{\max }=28 \mathrm{~nm}$ which is small enough to achieve a ductile mode material removal.

After cutting, a white light interferometer based optical surface profiler from Bruker cooperation (ContourGT-X8) as well as an atomic force microscope (AFM) from Oxford Instruments (MFP3D Infinity AFM) is adopted to capture features of the machined surface.

\subsection{Taper cutting based critical depth-of-cut determination}

To facilitate the toolpath determination, a set of taper cuttings are conducted to identify the critical depth-of-cut for different crystal orientations. From the optical microscope image in Fig. 4 (a), the cuttings are performed towards the center along different directions with an even angular interval of $15^{\circ}$. The critical depthof-cut is measured from the 3D topography of each generated channel which is measured by the optical surface profiler.

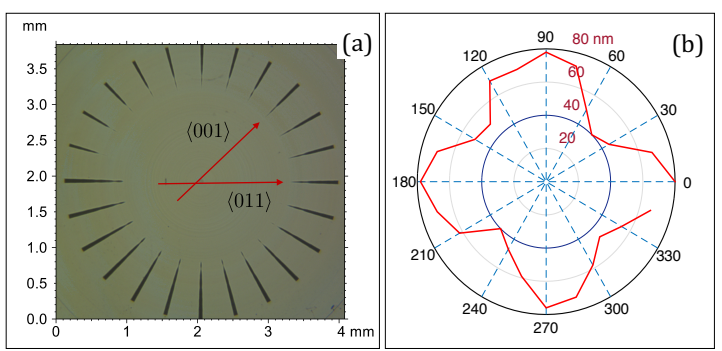

Figure 4. (a) the microscope image of the surface after taper cutting, and (b) the critical depth-of-cut with respect to the crystal orientations.

Accordingly, the identified critical depth-of-cut is depicted with respect to the crystal orientations in a polar coordinate system as shown in Fig. 4 (b). Overall, a quasi-periodic tendency is observed for the critical depth-of-cut, and the maximum and minimum value are about $80 \mathrm{~nm}$ and $40 \mathrm{~nm}$ which may correspond to the $\langle 011\rangle$ and $\langle 001\rangle$ direction, respectively. To avoid potential cracks for all cutting positions, the minimum value is chosen as the critical one for the whole surface, namely $h_{c}=40 \mathrm{~nm}$.

\subsection{Cutting performance demonstration}

As a demonstration, a typical micro-structured droplet surface described by $z_{s}=A_{s} \cos \left(2 \pi f_{s} \rho\right)+A_{s}$ is employed with $A_{s}=0.5 \mu \mathrm{m}$ and $f_{s}=8$. The nominal depth-of-cut is set as $h_{0}=2.5 \mu \mathrm{m}$, and the spindle speed is set as $1200 \mathrm{rpm}$. For the turning of such a droplet surface, the minimum allowable clearance is estimated to be $4.3^{\circ}$ from Eq. (6). Therefore, the selected cutting tool is capable to generate such a surface without any clearance interferences.
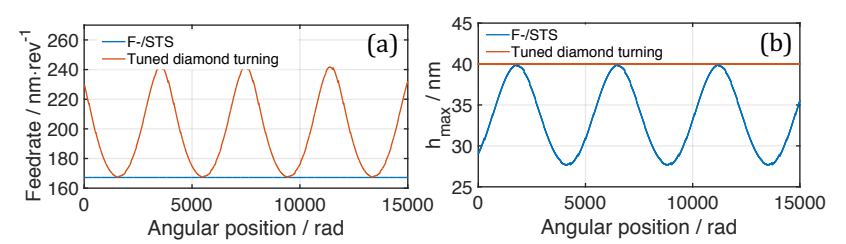

Figure 5. Feature comparison between the tuned diamond turning and F/STS, (a) the feedrate, and (b) the maximum depth-of-cut $\left(h_{\max }\right)$. 
With the tuned diamond turning, the feedrate $f_{k, l}$ is illustrated in Fig. 5 (a) with respect to the angular position of the spindle, which periodically varies between $167.2 \mathrm{~nm} / \mathrm{rev}$ and 241.7 $\mathrm{nm} / \mathrm{rev}$. Meanwhile, the maximum allowable feedrate for F-/STS is restricted to be constant as $167.2 \mathrm{~nm} / \mathrm{rev}$ to avoid cracks (Fig. 5 (a)). As illustrated in Fig. 5 (b), attributing to the constant feedrate in F-/STS, the maximum depth-of-cut exhibits a high dependence on the angular position which is closely related to the remote shape of the desired surface. By contrast, taking advantage of the tuning concept, a constant maximum depth-ofcut is achieved as desired, suggesting that a much higher material removal rate can be obtained for the tuned diamond turning.

To have a quantitative comparison of the machining efficiency, the required numbers of spindle revolutions for F-/STS and the tuned diamond turning of an area with a radius of $0.7688 \mathrm{~mm}$ are further checked to be 4598 and 3846, respectively. It suggests that compared to F-/STS, the machining efficiency is improved by a factor of $16.35 \%$ by adopting the tuned diamond turning.

To tune for the droplet surface, an oscillation along the $x$-axial direction of the machine tool (Fig. 6 (a)) is superimposed on the conventional linear radial motion. In addition, the trajectory tracking performances for the DA-FTS along the $x$ - and $z$-axial directions are given in Figs. 6 (a) and (b), respectively. A good agreement between the desired and practical motion is observed for each moving axis. The tracking error in Fig. 6 (c) shows no obvious tendency components, and the maximum error for both axes is down to the level of system noises $( \pm 5 \mathrm{~nm})$. It suggests that the developed DA-FTS is of sufficient tracking accuracy to perform the tuned diamond turning.
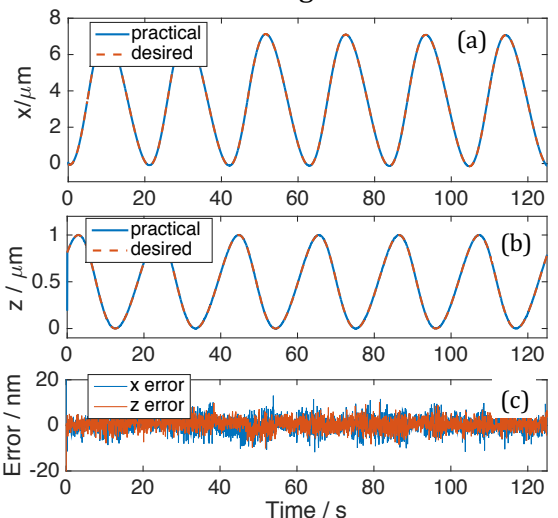

Figure 6. The trajectory tracking performance, (a) the $x$-axial direction, (b) the $z$-axial direction, and (c) the corresponding tracking errors.

\subsection{Quality of the machined surface}

After turning, the optical microscope image of the whole cutting region is captured as shown in Fig. 7 (a), where a mirror surface without obvious cracks and defects is observed. By means of the surface profiler, the 3D micro-topography of a region along the $\langle 001\rangle$ direction is presented in Fig. 7 (b). The cross-sectional profile along the radial direction is shown in Fig. 7 (c), together with the desired one and the deviation between the two profiles. In general, the deviation suggests that the maximum form error as obtained is within $\pm 89.5 \mathrm{~nm}$. Considering the small tracking errors (less than $10 \mathrm{~nm}$ ) for the dual axes in Fig. 6, the form error might be jointly caused by the error motions of the machine tool and the possible parasitic motions of the DA-FTS.

Since the feedrate is much smaller than the wavelength of the optical microscope, the AFM is employed to capture more detailed features of the machined surface with a sub-wavelength lateral resolution. The measured area $(2 \mu \mathrm{m} \times 2 \mu \mathrm{m})$ in Fig. 7 (d) exhibits a clear view of the uniformly distributed tool marks which have similar features of the tool marks obtained in the turned metallic surfaces. Moreover, the surface roughness of this area is statistically calculated to be $S a=1.18 \mathrm{~nm}$. The obtained result suggests that a ductile mode removal of the material is achieved for the tuned diamond turning.
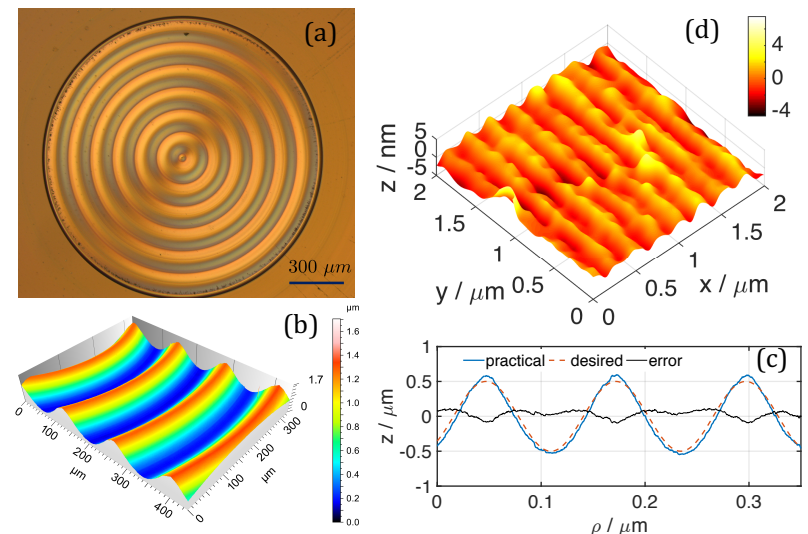

Figure 7. Characteristics of the generated surface, (a) the optical microscope image, (b) the 3D micro-topography of a smaller region, (c) the radial cross-sectional profile, and (d) an AFM image of the surface.

\section{Conclusion}

To improve the machining efficiency for the turning of microstructured surfaces on brittle materials, a novel tuned diamond turning technique is proposed with the maximum depth-of-cut being strictly consistent with the critical one of the workpiece material. To tune the depth-of-cut, an extra-oscillation along the $x$-axial direction is introduced to closely cooperate with the $z$ axial oscillation in conventional fast-/slow tool servo. Taking advantage of a newly developed dual-axial fast tool servo, a crack free micro-structured surface is obtained on a single crystal silicon wafer with the machining efficiency improved by a factor of $16.35 \%$, and the profile error and surface area roughness ( $\mathrm{Sa}$ ) are obtained to be less than $\pm 90 \mathrm{~nm}$ and $1.2 \mathrm{~nm}$, respectively.

\section{Acknowledgments}

This work was supported by the National Natural Science Foundation of China (51705254, 51675455), and the General Research Fund from the Research Grants Council of Hong Kong SAR (PolyU 152021/17E). Z. Zhu would also thank the financial support from the Alexander von Homboldt Foundation, Germany.

\section{References}

[1] Mukaida M and Yan J (2017). Ductile machining of single-crystal silicon for microlens arrays by ultraprecision diamond turning using a slow tool servo. International Journal of Machine Tools and Manufacture, 115, 2-14.

[2] Yan J, Zhang Z, and Kuriyagawa T (2009). Mechanism for material removal in diamond turning of reaction-bonded silicon carbide. International Journal of Machine Tools and Manufacture, 49(5), 366-374.

[3] Fang FZ, and Chen LJ (2000). Ultra-precision cutting for ZKN7 glass. CIRP Annals, 49(1), 17-20.

[4] Yu DP, Wong YS, Hong GS (2011). Ultraprecision machining of micro-structured functional surfaces on brittle materials. Journal of Micromechanics and Microengineering, 21(9), 095011.

[5] Owen JD, Troutman JR, Harriman TA, Zare A, Wang YQ Lucca DA, Davies MA (2016). The mechanics of milling of germanium for IR applications. CIRP Annals, 65(1), 109-112.

[6] Fang F, Chen Y, Zhang X, Hu X, Zhang G (2011). Nanometric cutting of single crystal silicon surfaces modified by ion implantation. CIRP Annals, 60(1), 527-530.

[7] Mohammadi H, and Patten JA (2017). Effect of thermal softening on anisotropy and ductile mode cutting of sapphire using micro-laser assisted machining. Journal of Micro and Nano-Manufacturing, 5(1), 011007.

[8] Suzuki N, Haritani M, Yang JB, Hino R, Shamoto E (2007). Elliptical vibration cutting of tungsten alloy molds for optical glass parts. CIRP Annals, 56(1), 127-130. [9] Zhu Z, Zhou X, Liu Z, Wang R, Zhu L (2014). Development of a piezoelectrically actuated two-degree-of-freedom fast tool servo with decoupled motions for micro/nanomachining. Precision Engineering, 38(4), 809-820.

[10] Yang X, Liu H, Xiao J, Zhu W, Liu Q, Gong G, Huang T (2018). Continuous Friction Feedforward Sliding Mode Controller for a TriMule Hybrid Robot. IEEE/ASME Transactions on Mechatronics, 23(4), 1673-1683. 